\title{
A practical guide to effective behavior change: how to identify what to change in the first place
}

Gjalt-Jorn Ygram Peters

This document is the full text of the article "A practical guide to effective behavior change: how to identify what to change in the first place" that has been published in the European Health Psychologist in 2014.

Although the European Health Psychologist is Open Access, it does not associate digital object identifiers to its publications. PsyArXiv does associate DOI's to the posted preprints, and in addition, PsyArXiv preprints are widely indexed. Therefore, this version has been published on PsyArXiv as well.

\section{Citing this manuscript}

To cite this manuscript, use the following citation:

Peters, G.-J. Y. (2014). A practical guide to effective behavior change: how to identify what to change in the first place. European Health Psychologist, 16(4), 142-155. doi:10.31234/osf.io/hy7mj

\section{Other behaviour change resources}

A number of behaviour change resources that may be useful to intervention developer or behaviour change researchers are available at https://effectivebehaviorchange.eu. In addition, the website of the Academy of Behavior Change, https://a-bc.eu, may be useful.

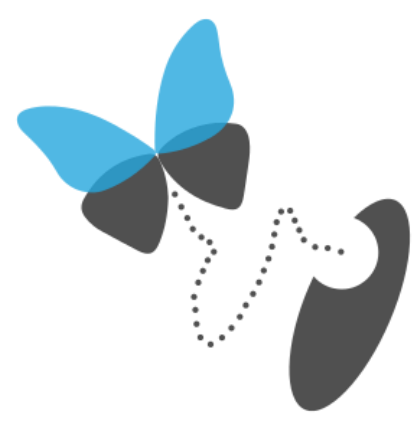




\section{A practical guide to effective behavior change: How to identify what to change in the first place}

Gjalt-Jorn Y. Peters In 1947, mathematician Open University of The John von Neuman Netherlands

supporting this claim with a comparison with something infinitely more complicated: "If people do not believe that mathematics is simple, it is only because they do not realize how complicated life is" (Alt, 1972). Behavior change scientists who study behavior change or who develop behavior change interventions operate in between these two extremes of overwhelming complexity and, well, at least relative simplicity. Effective behavior change methods (Peters, de Bruin, \& Crutzen, 2014) employ theorybased ${ }^{1}$ processes of change to influence psychological variables that are postulated to determine behavior. Because, bar physical coercion, no behavior change method operates directly on behavior, this means that identifying the relevant determinants and beliefs to influence is a crucial step in the development, or evaluation, of any behavior change method/technique. The current contribution intends to pave the way for more in-depth discussion of behavior change by outlining basic guidelines for establishing which determinants, and, maybe even more importantly, which beliefs, to influence. This document has been set up to be useful when explaining the basics of behavior change to, for example, students, early-career Ph.D. candidates, or practitioners. Therefore, it will start with outlining, assuming very little knowledge of psychology, why it is imperative to map beliefs and determinants before

1 After all, even basing method employment on exclusively empirical evidence in itself constitutes implicit postulation of a theory regarding the determinants/beliefs that method targets and under which conditions; this implicit theory will just be excessively simplistic in most cases. even thinking about how to change a given behavior. In the second part, practical pointers will be given as to how to actually do this - map beliefs and determinants. In other words, first I'll explain what to change; and then, how to identify what to change.

\section{Starting from scratch}

Although the approach outlined in these guidelines is not based on any particular single theory, it does make a number of basic assumptions. To make sure that everybody is on the same page, and to make this text as widely accessible as possible, I will start with outlining these assumptions, so feel free to skip the next paragraphs if you already know all this (this bit is where the pretty pictures are though). The first assumption is that influences on human behavior exist either inside or outside a person. Influences outside a person are discussed below in the section on environmental conditions. The second assumption specifically concerns influences within a person. These influences are not directly observable (skin, bones, and muscle tissue unfortunately block the view), so psychologists resort to a variety of indirect methods and combine these with sets of assumptions to draw conclusions about a person's psyche. For example, some methods detect electrical signals or oxygen transport and assume that these are correlated to psychological activity. Other methods use questionnaires, assuming that the answer options that participants endorse provide data on their psyche; or computer tasks measuring reaction times, assuming that comparison of different types of reaction times provides information about associations within participants' psyches. 
One assumption, for which a lot of evidence has been collected, is the assumption that human sensation, emotion, and cognition (basically, everything determining behavior) consists of activation patterns of neurons ('brain cells'). At any given moment, each neuron has an activation level. If this activation level reaches a certain threshold, the neuron 'fires': this causes it to release molecules (called neurotransmitters) that either increase or decrease the activation levels of whichever neurons the firing neuron is connected to. Figure 1 shows an example of three neurons. If the activation level of neuron 1 reaches its threshold, it will activate (or potentially inhibit) neurons 2 and 3 . If such activation ('excitation', technically) causes the activation levels of neuron 2 to reach the threshold as well, neuron 2 will also release neurotransmitters to contribute to the excitation or inhibition of neuron 3 , which may then in turn excite or inhibit more neurons. Perception of external stimuli (a sunrise, the smell of bacon, or the touch of a friend) cause the activation of neurons; motor activity (basically, any observable behavior) is controlled by activation of neurons; and in between, a lot of neurons are excited and inhibited (around 86 billion; Azevedo et al., 2009). Thus, at the most fundamental level, it appears that the human psyche consists of, or functions through, neurons that activate and inhibit each other.

Interestingly, these spreading activation patterns as illustrated in Figure 1 have been shown to exist at higher conceptual levels, as well. An example I frequently use in presentations and trainings for practitioners is the DRM paradigm (Roediger \& McDermott, 1995). This is a simple paradigm for creating a very basic 'false memory'. Participants are read a list of words and are instructed to remember these. Then, another word list is read aloud and participants have to indicate, for each word, whether it was present in the first list. The words on the first list are all strongly related to one core concept, such as 'sleep', which itself is not on the list. These relationships, however, all cause the core concept to

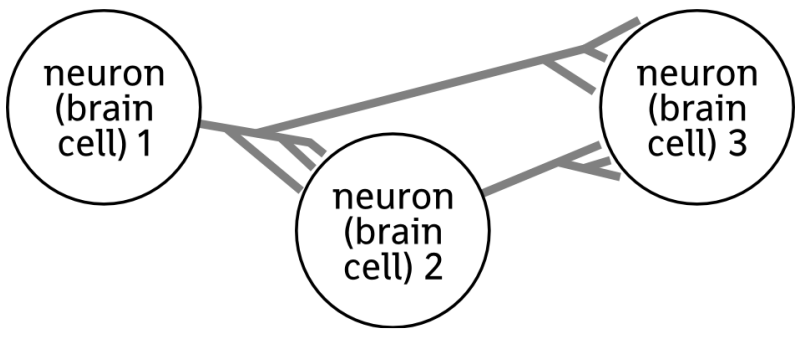

Figure 1: Three connected neurons.

become slightly more activated as more and more related words are presented, and when the core concept is finally listed in the second list, many participants erroneously indicate that they heard it in the first list (when asking a group to raise hands, around one-thirds indicate this, in my experience). Figure 2 shows a fragment of this paradigm; through their connections, 'bed' and 'rest' each contribute to the activation levels of 'sleep'. Of course, more everyday examples are available as well: one memory can trigger another; the first movement in the procedure of tying ones shoelaces automatically activates the next; and more relevantly, pairing cues and thoughts such as when using implementation intentions (see Hagger \& Luszczynska, 2014, this issue) can cause perception of a mailbox to remind one of mailing a letter. Thus, it appears this concept of spreading activation is a useful metaphor when thinking about explaining and changing human behavior.

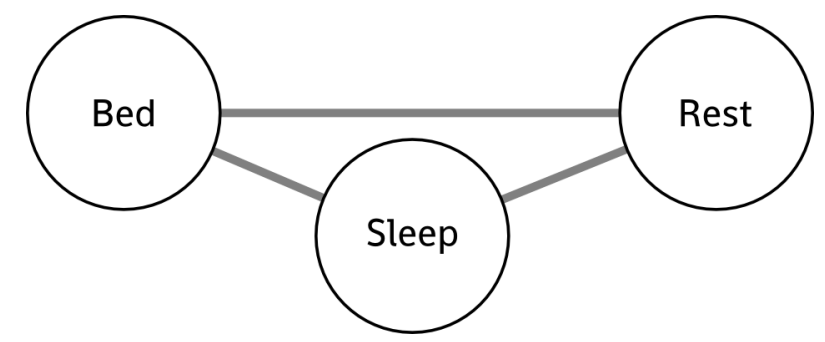

Figure 2: Three connected words or concepts.

\section{What to change: determinants and beliefs}

Now, back to determinants and beliefs. The guidelines outlined in the present contribution are 


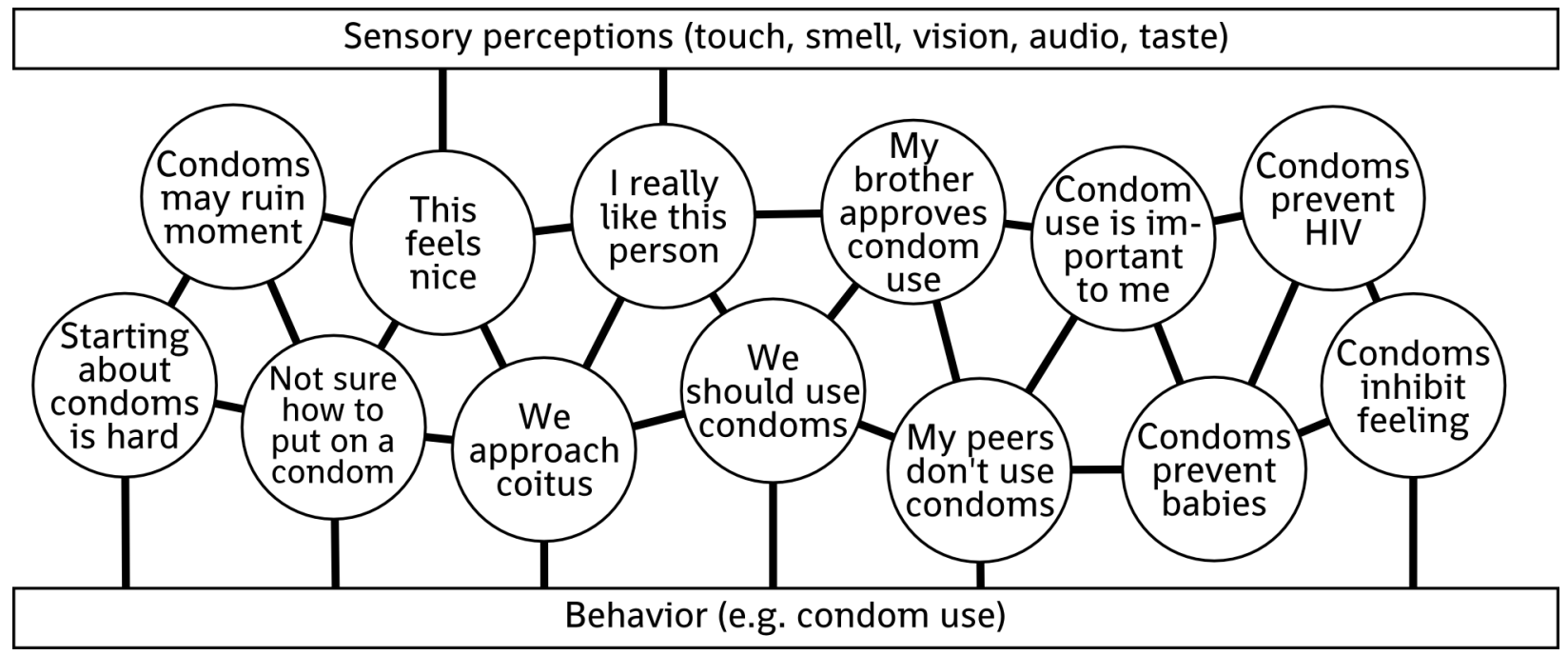

Figure 3: Connected beliefs that contribute to the decision to use condoms.

based on the assumption that behavior is a consequence of a set of processes operating on a set of variables. These variables can be visualised as a network of interconnected nodes, each node representing, for example, a perception, emotion, or cognition. An example showing a fragment of such a network involved in the decision to use condoms during sexual intercourse is shown in Figure 3. Each node corresponds to a belief. It is important to note two things. First, of course, such beliefs do not actually exist in our brains; this visualisation is just a useful metaphor that captures some of the properties of beliefs, which helps to think about beliefs and determinants. Second, although so-called 'implicit' cognitions (as opposed to the more easily verbalisable 'explicit' cognitions), as well as processes that operate on these variables, have been omitted from this illustration, this metaphor holds for those as well. The basic idea is: there is stuff in our minds; this stuff is connected; and when discussing this stuff, it is useful to distinguish entities we call 'beliefs', which correspond to single thoughts, emotional associations, perceptions, cognitions, elements of processes, concepts, associations between

2 As I said, this redefinition of a belief as a specific, bounded psychological entity, be it a cognition, an affective association, or an element of a process, is much broader than the definition usually used in the literature. concepts, etc. Note that this definition of a belief as a psychological entity is substantially broader than the definition generally used in the literature, for example in the Reasoned Action Approach (Fishbein \& Ajzen, 2010). In this shiny new definition (i.e. as a psychological entity that is a component of a determinant), a 'belief' can also be an implicit association, or an element of a process, such as attention deployment. This is useful, because in this new definition, all human behavior within a given environment is (by definition) determined by beliefs ${ }^{2}$. It follows that mapping all beliefs allows prediction of behavior.

However, the low-level, specific nature of these beliefs also means that they have a very narrow scope. The belief that condoms prevent HIV will likely contribute to some extent to the decision to use condoms during intercourse; its role in the decision to go jogging despite the rain is likely considerably less substantial (of course, the connections of the belief that condoms prevent HIV to beliefs related to jogging and rain are also very weak or non-existent). Fortunately, psychologists have identified categories of functionally similar or functionally related lowerlevel psychological entities (beliefs). Such categories are higher-level variables: compound constructs that aggregate these functionally similar or functionally 


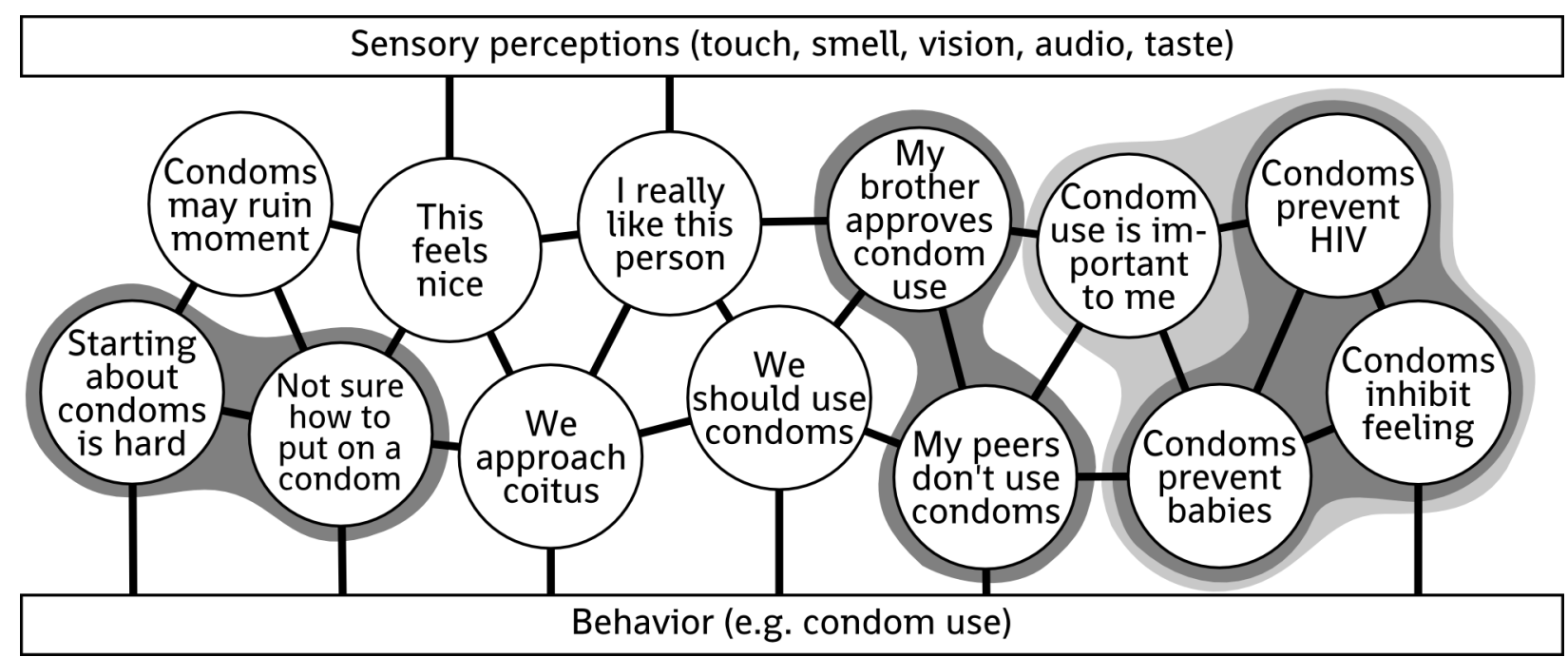

Figure 4: Connected beliefs that contribute to the decision to use condoms, with the determinants formed by the beliefs indicated by shading (from left to right, self-efficacy, subjective norm, traditional TPB attitude (dark gray) and revised RAA attitude including 'importance' (light gray)).

related lower-level beliefs. The definitions of these compound constructs are formulated in theories, which I here consider to be anything that combines variables (something which can have a value) and processes (something which changes values of variables) in a hopefully somewhat coherent description of how part of the human psyche operates. Well-known examples in health psychology are the Reasoned Action Approach (Fishbein \& Ajzen, 2010), Social Cognitive Theory (Bandura, 1991), the Health Action Process Approach (Schwarzer, 2008), and the theory of Implementation Intentions (Gollwitzer, 1999; Sniehotta, Schwarzer, Scholz, \& Schüz, 2005). Psychological theories postulate psychological variables that predict behavior (or other variables) and/or postulate processes that explain the dynamics of these variables, such as processes by which psychological variables can be changed. The Reasoned Action Approach, for example, mainly concerns itself with defining a number of determinants of behavior and how they relate to each other, rather than postulating methods to change these determinants ${ }^{3}$; Social Cognitive

3 Although the RAA explains that changing a determinant requires changing the underlying beliefs, it does not concern itself with methods to change those beliefs and thus the overarching determinants.
Theory, on the other hand, does postulate such methods, such as Modeling. Theories about behavior change are discussed more in depth in the contribution by Kok (2014, this issue); for now, we focus on theories that explain behavior.

When a theory postulates the existence of one or more psychological variables, it frequently also defines the (kind of) beliefs that together form the hypothesized variables(s). After all, if the theory limits itself to the description of one or more psychological variables as abstract entities, without considering operationalisation of those variables, the theory cannot be studied empirically. For all practical purposes a psychological variable is its operationalisation; any aspects of a variable that are not measured or manipulated unfortunately but necessarily fall without the scope of empirical investigation. If a theory does provide an operationalisation (i.e. a measurement instrument, manipulation, stimulus, etc) or guidelines detailing how to develop an operationalisation, this operationaliation, or the guidelines, will have to be somewhat specific and concrete: in other words, these operationalisations or guidelines address beliefs. For example, questionnaires measuring psychological variables necessarily use relatively concrete/specific items to make sure that all participants can complete 
the questionnaire: the variation in capacity for abstract thought in most populations means that using items that employ complex, abstract, concepts severely undermines the validity of the relevant measure.

Sticking to the 'activation patterns' metaphor of beliefs and determinants, a theory, then, consists of definitions as to which beliefs together form which psychological variables. This is illustrated in Figure 4. The RAA, for example, holds that the impression that starting a conversation about condoms is hard and uncertainty about how to put on a condom would together (with many more beliefs) form ${ }^{4}$ the determinant 'perceived behavioral control'. Similarly, the RAA holds that the determinant called 'perceived norm' consists of beliefs such as the perceptions that ones brother approves of condom use, and that ones peers do not use condoms; and that the beliefs that condom use prevents HIV and pregnancy, and that condoms decrease the sensations during intercourse together form 'attitude'. Different theories can (and, of course, often do) contain contradictory definitions. For example, the Reasoned Action Approach holds that effects of distal behavioral predictors such as personality, past behavior, age and gender are mediated by the more proximal determinants attitude, subjective norm, and perceived behavioral control, which in turn predict the even more proximal determinant intention, which finally predicts behavior. However, over time, several potential fourth proximal determinants have been proposed, such as self-identity. One belief proposed to be a part of selfidentity is "Condom use is important to me". In their 2010 revision of the TPB into the RAA, Fishbein and Ajzen argued that in fact, this aspect of self-identity can be subsumed in their attitude construct, when a number of adjectives assessing the importance of a

\footnotetext{
4 Note that in most situations, it is very hard to empirically distinguish whether two psychological variables influence a third variable, or whether the two variables are a part of the third variable. In both cases, the same pattern of correlations will be observed, and in both cases, changing one of the two 'minor' variables changes the 'major' variable. Neither case usually makes explicit predictions as to what happens with the 'minor' variables if the 'major' variable is changed.
}

behavior are added to the semantic differentials used to operationalise attitude (2010, p. 292). Of course, when trying to explain behavior, the most important thing is that all relevant beliefs are identified, and of which particular psychological variable one considers these beliefs to be a part is secondary. However, when trying to change behavior, determining to which determinant a belief 'belongs' becomes crucial, because methods for behavior change are usually matched to determinants, not to beliefs. After all, psychological researchers usually try to study behavior change methods that are applicable across a variety of behaviors and populations. Applying a behavior change method that has been shown to successfully enhance self-efficacy, such as Guided Practice, to change a belief that doesn't underlie selfefficacy but instead underlies subjective norm, such as "My parents disapprove of me drinking too much", will most likely fail. Therefore, it is important to have a clear idea of which beliefs 'belong' to which determinants (and therefore, often, which theories are employed).

\section{What to change: environmental influences on behavior}

In addition to influences within a person, there are also influences in a person's environment that influence his or her behavior. Free provision of condoms facilitates condom use; ubiquity of gyms facilitates exercise; and widespread availability of unhealthy foods complicates adherence to a healthy diet. These environmental conditions are usually changeable as well - they can usually be changed by the behavior of other people. Such other people, holding key positions in the environment of target population individuals, are usually referred to as environmental agents or actors (Kok, Gottlieb, Commers, \& Smerecnik, 2008). Examples are parents, partners, nurses, teachers, directors of hospitals, school boards, or politicians. Each environmental 
actor is located with a certain proximity to the target population, or perhaps more accurately, has a certain role in the environment of the target population. For example, some environmental agents directly socially interact with the target population; others control an infrastructure in which the target population is involved either directly (employers, schools, hospitals) or indirectly (politicians). Four such environmental levels are often distinguished, usually visualised as concentric circles around the target population individuals: interpersonal (e.g. parents, peers), organisational (e.g. director, school board), communal (e.g. community leaders, religious leaders), and societal (e.g. politicians). In theory, a fifth 'global' level exists (e.g. the World Health Organisation and the United Nations). These levels are useful, because research into behavior change methods to target environmental actors has identified, for example, methods that work for actors on the societal level, methods that work for actors on the organisational level, etc. The contribution by Kok (2014, this issue) will treat this more thoroughly: for now, it is important to remember that environmental influences on behavior can be traced to environmental agents, and that for each of these agents, it is important to identify on which environmental level(s) they reside.

\section{What to change: behaviors and sub- behaviors}

There is one more important term to agree on before starting with the practical guidelines. This relates to sub-behaviors. When we discuss behaviors we want to change, we often talk about very broadly defined behaviors, such as smoking, safe sex, physical activity, substance use, or diet. These behaviors usually comprise a set of preparatory and/or subbehaviors, sometimes quite limited and clear (van Empelen \& Kok, 2006), but sometimes compiling an exhaustive list can be practically impossible (e.g. diet). In all cases, it is important to try to be aware of which preparatory and/of sub-behaviors are to be influenced, because the determinants and environmental conditions can differ between these behaviors. For example, adolescents may have different reasons to refrain from buying condoms than they have for carrying condoms; and different reasons again for bringing up the subject of condom use with a partner. Making these preparatory and/or sub-behaviors explicit makes it much easier to obtain an overview of the relevant determinants and environmental conditions. We could say that any behavior change intervention in fact has several behavioral objectives, each of which concerns performance of a preparatory or sub-behavior. Therefore, from here on we will refer to such preparatory and/or sub-behaviors as performance objectives of an intervention (Bartholomew, Parcel, Kok, Gottlieb, \& Fernández, 2011). Achieving all performance objectives, then, means that the intervention is effective; if all performance objectives are performed, the overarching behavior is by definition also performed. If one or more performance objectives are not achieved, the likelihood that the overarching behavior is performed is much lower; for example, if an adolescent buys condoms, but does not carry condoms when needed, the likelihood of safe intercourse is very low. Thus, an intervention promoting condom use that addresses communication about condoms is more likely to be effective than an intervention that only addresses the benefits of condom use.

\section{How to identify what to change in the first place}

Combining these bits of information, we could say that most behaviors consist of sub-behaviors (called performance objectives in a behavior-change context), each of which is determined by personal determinants and environmental conditions. We have 
psychological theories proposing ways in which these determinants are related to each other and to behavior, as well as theories about how to change these variables. Because determinants have similar dynamics over behaviors and populations, they are by definition generic and abstract: determinants are convenient categories of functionally similar or functionally related sub-entities which we call beliefs. These beliefs are specific to behaviors and populations, and therefore provide 'tangible', concrete objectives to target in an intervention. Now a vocabulary has been established, we can discuss the task of identifying these performance objectives, environmental conditions, determinants, and beliefs for a given target behavior. In applied research such as this, methodological promiscuity has considerably benefits. Ideally, an overview of the existing literature is supplemented with interviews with target population members and possibly key environmental actors, and the results of these two steps are quantitatively verified so that the relative importance of determinants and beliefs can be established. Each of these three steps will be explained below in more detail.

\section{Systematic reviews}

A useful start is to compile the available empirical evidence. It usually pays to do this sufficiently thorough and systematic, so that the findings can be published in the literature. That way, others can benefit from your efforts as well. There are a lot of workshops and resources available that deal with conducting systematic reviews (Khan, Kunz, Kleijnen, \& Antes, 2003; Moher, Liberati, Tetzlaff, \& Altman, 2009), but many of these focus on how to extract, analyse, and report data. Therefore, to give you an idea of all involved phases, Box 1 shows a rough overview in case you want to plan a systematic review.

Ideally, there is a lot of literature available reporting correlations between determinants and behavior, or between more distal determinants of behavior, such as self-efficacy, and more proximal determinants of behavior, such as intention. In such situations a meta-analysis can be conducted to integrate the evidence. Sometimes, there is even enough evidence to examine which beliefs are important in the same way. Often, however, a lot of evidence, especially on the more specific level of beliefs, will be qualitative; and sometimes, bivariate associations are not reported, and only univariate results such as percentages of participants endorsing a belief are reported. Such evidence is by design excluded from a meta-analysis, and therefore, it is usually worthwhile to consider conducting a qualitative review as well. For an example of a metaanalysis and subsequent qualitative analysis on the same topic, see Peters, Abraham and Kok (2008) and Peters and Kok (2009).

A literature overview and integration yields an overview of what is already known about your target behavior, sometimes even for your target population. However, what is known might be very little; and even if it is a lot, it is very common that most studies examined populations that differ slightly, or a lot, from the target population at hand. Therefore, it is often necessary to verify these findings for the specific target population and context at hand.

\section{Interviews}

Whenever you develop an intervention, it pays to actually talk to your target population members and relevant stakeholders. In fact, it is wise to involve them in an early stage, for example in so-called Linkage Groups (Bartholomew et al., 2011). However, in addition to their active participation in the intervention development and preparation for that development, interviewing target population members in a qualitative study also has substantial benefits. Any behavior change intervention that does not exclusively work through environmental change will need to know which beliefs are important to the 


\section{Box 1: Basic steps for synthesizing the literature on determinants and beliefs}

1. On the basis of your research question and your knowledge of the literature, develop a first version of a coding sheet to extract methodological and statistical data from publications;

2. On the basis of your research question and the information required in your coding sheet, establish in- and exclusion criteria, and invert all inclusion criteria to exclusion criteria that can be used for screening;

3. On the basis of your research question, coding sheet, and in- and exclusion criteria, craft a query using the logical operators OR (to combine synonymous keywords) and AND (to combine sets of synonymous keywords);

4. Select bibliographic databases and interfaces to use (e.g. PubMed using its own interface; PsycINFO using Ebscohost or Ovid; etc), and translate your query to each database/interface combination;

5. Run the query in all interfaces, export the hits and merge these records into one file;

6. Establish exclusion criteria and let two or more independent screeners screen the records on basis of title/abstract;

7. Acquire full texts for all records that could not be excluded by both screeners, and let the screeners screen these again in the second screening round;

8. Apply secondary methods to identify relevant publications, such as the ascendancy approach (screen reference lists of included publications), descendancy approach (screen publications citing the included publications), and identifying grey literature (e.g. through mailing lists etc);

9. Use the coding sheets to extract methodological and statistical data;

10. Synthesize the data and report your results.

NOTE: the appendix at http://osf.io/fp8kv has a list of free and cheap software packages that you can use for these tasks.

target population. After all, even if the important determinants have been identified, any intervention targeting these determinants will need to communicate one or several specific messages, and these messages need to address beliefs that are important to the targeted determinants. For some behaviors, risk perception beliefs will be an important part of attitude; for other behaviors, risk perception will be irrelevant; and even if risk perception beliefs are important, the risks that are perceived differ from behavior to behavior and from population to population. Therefore, conducting a qualitative study can considerably increase the likelihood that your intervention is effective. Like for systematic reviews, the basic steps in conducting a qualitative study are summarized (see Box 2; and for an excellent practical textbook, see e.g. Ritchie, Lewis, McNaughton Nicholls, \& Ormston, 2014).

At this point, you will have collected a wealth of information about why your target population behaves the way they do. You will know the reasons that people report for behaving as desired, and the reasons that people report when they exhibit the undesirable behavior (usually the unhealthy behavior). You will know what sub-behaviors people consider this behavior to consist of, and you will know which environmental conditions, beliefs, and determinants might play a role for each sub-behavior. Unfortunately, 'might' is the operative word here. After all, people are not always aware of the reasons 


\section{Box 2: Basic steps for qualitative exploration of determinants and beliefs}

1. On the basis of the literature review, theory, and consultation with experts regarding your target behavior and target population, establish a topic list of topics that you want to address in the interviews. Make sure that you also pay attention to what participants actually do: your definition of the behavior at hand might differ from theirs;

2. Determine whether you will only conduct individual interviews, or also focus groups. Focus groups allow observation of norms and group dynamics, but can inhibit openness of individual participants. For public behaviors, focus groups can be a useful addition, whereas for more private behaviors, they may have little added value (and be quite awkward);

3. Recruit target population members. This may be quite hard depending on your target population; when you study exercise behavior among students, it's considerably easier than when you are interested in needle sharing among hiv-positive migrant sex workers. Getting in touch with dedicated NGO's may be very helpful (in fact, having these in your Linkage Group can be helpful as well);

4. Secure a quiet, neutral venue, recording equipment, and possibly organise support for the interviewer and/or the participants (interviews can become quite intense). Also, acquire ethical approval and plan your data management (e.g. how will you make sure the original audio recordings are safely stored and that only one or a few people have access?);

5. Conduct the interviews, updating the topic list as your insights develop;

6. Transcribe the data (or get an organisation to transcribe; this may be considerably cheaper);

7. Code the data. Specifically, try to identify whether the beliefs you observe 'belong to' a known determinant. Our knowledge on methods for change is, after all, based on research into determinants.

8. Report your results.

NOTE: the appendix at http://osf.io/fp8kv has a list of free and cheap software packages that you can use for these tasks.

for their behavior. In addition, reasons that are considered important to people do not necessarily have to be important predictors of behavior: after all, the correlation of a belief with behavior is rarely a factor people take into account when determining how important they consider the belief. Therefore, a quantitative verification of the combined outcome of the literature review and the qualitative study is often necessary.

\section{Survey}

As De Bruin, Viechtbauer, Hospers, Schaalma and Kok concisely stated, "the quality of an intervention can be defined as the degree to which effective behavior change techniques are adequately applied to important determinants of target behaviors" (2009), and this means that determining relative importance of behavioral determinants is necessary. By extension, given that any behavior change method will have to address specific beliefs, this means that determining relative importance of the beliefs underlying each determinant is also necessary. This requires 


\section{Box 3: Basic steps for quantitative verification of determinants and beliefs}

1. Compile an overview of all determinants and beliefs that you identified up until this point;

2. Develop operationalizations for each determinant and belief. Some theories provide guidelines; Francis et al. have developed an excellent manual for the TPB (2004), and the RAA (Fishbein \& Ajzen, 2010) also contains sections on measurement. In any case, try to avoid categorical or dichotomous operationalisations, as these considerably decrease your power and are usually less valid. Avoiding 'disagree-agree' answer options can also be useful, as 'disagree' can often be interpreted either as "neutral" or "the opposite";

3. Combine these operationalizations in one measurement instrument, and pilot-test this with your target population to make sure your items are understandably and unequivocally formulated;

4. Acquire ethical approval and plan your data and resource management (e.g. if your data is not collected anonymously, how will you anonymize it, and how will you restrict access to the raw, un-anonymized datafiles? If you do collect your data anonymously, then how will you obtain the measures of participants' behavior(s) after the chosen timeframe? How will you secure your resources for later inspection and publication?);

5. Once you have your data, visualise the univariate distributions of all variables, inspect scattermatrices to assess item and variable associations, and aggregate items into variables (see e.g. Peters, 2014).

6. Compute confidence intervals for correlation coefficients to estimate how strongly each determinant predicts your target behavior or performance objective (the 'userfriendlyscience' $R$ package described in Peters (2014) contains the function rMatrix, which creates a correlation matrix with confidence intervals);

7. Compute confidence intervals for correlation coefficients to estimate how strongly each belief predicts the determinant it is a part of;

8. Conduct a regression analysis to obtain an $R^{2}$ measure to get an impression of the degree to which you understand your performance objective or target behavior and each respective determinant. Note that regression coefficients should not be interpreted as coefficients of importance; bivariate analyses lend themselves better to determine relative importance.

9. Report your results.

NOTE: the appendix at http://osf.io/fp8kv has a list of free and cheap software packages that you can use for these tasks.

quantitative data, which can be acquired using, for example, a survey5. Box 3 shows the steps typically involved in conducting such a survey.

5 Of course, if methods are available to change implicit associations, it can be useful to measure these as well; and similarly, measuring processes, such as for sample self-regulation, can be useful as well.

\section{Towards selection of behavior change methods/techniques}

After these three studies, you will have a pretty good idea of what you should target in your intervention. At this point, it is easy to get quite confused by the overwhelming plethora of performance objectives, determinants, and beliefs. 
Fortunately, there exists a standardised method of combining and documenting all this information for a given behavior and population: the so-called matrix of change objectives (Bartholomew et al., 2011). Such a matrix provides a convenient method to organise almost everything you know about predicting a given behavior. The rows of the matrix are the performance objectives (the preparatory/sub-behaviors), and the columns are the determinants. In each cell, the beliefs are listed for the corresponding determinant/performance objective combination. Of course, some cells will contain multiple beliefs and some will be empty. It can be convenient (and is somewhat customary) to phrase these beliefs as socalled 'change objectives', describing the desirable situation to be achieved. For example, the control belief related to one's ability to go to the gym in adverse weather conditions, which might be measured

\begin{tabular}{|c|c|c|c|}
\hline $\begin{array}{l}\text { Target population: EHPS member } \\
\text { Target behavior: submit EHP } \\
\text { contribution }\end{array}$ & Determinant 1: Attitude & Determinant 2: Injunctive norm & Determinant 3: Self-efficacy \\
\hline P01: Decide to contribute & $\begin{array}{l}\text { C01.1.1: explains how } \\
\text { contributing can foster } \\
\text { collaboration and networking } \\
\text { CO1.1.2: explains how contributing } \\
\text { can cause a warm fuzzy feeling }\end{array}$ & $\begin{array}{l}\text { C01.2.1: describes peers' } \\
\text { approval of EHP contributions }\end{array}$ & \\
\hline P02: Select an interesting topic & & $\begin{array}{l}\text { C02.2.1: describes peers as being } \\
\text { interested in interesting topics }\end{array}$ & $\begin{array}{l}\text { CO2.3.1: expresses confidence in } \\
\text { determining what topics are 'hot' } \\
\text { in the field } \\
\text { CO2.3.2: expresses confidence in } \\
\text { identifying an intersection of } \\
\text { these 'hot' topics with one's own } \\
\text { expertise }\end{array}$ \\
\hline P03: Approach co-authors & $\begin{array}{l}\text { C03.1.1: explains how writing } \\
\text { papers alone can feel lonely }\end{array}$ & & $\begin{array}{l}\text { C03.3.1: explains how to } \\
\text { convince co-authors of the } \\
\text { benefits of collaborating on an } \\
\text { EHP contribution }\end{array}$ \\
\hline
\end{tabular}

Figure 5: The matrix of change objectives for a target population individual, and at the organisation environmental level, an actor with his/her matrix of change objectives to change an environmental condition. 
with the item "I know I can go to the gym, even if it rains" in a questionnaire, corresponds to the change objective "The target population individual expresses confidence regarding going to the gym despite rainy weather". If this change objective has been achieved, the likelihood that the target population individual exercises regularly is increased. So, if all change objectives for a performance objective have been achieved, the performance objective has been achieved; and if all performance objectives have been achieved, the relevant individual changed his or her behavior, at least, to the degree that this behavior is independent from environmental conditions. To change these environmental conditions, it is necessary to identify which environmental agents have the ability to change these conditions, and then identify what they can do to effect these changes. of course, these environmental agents' behaviors consist of performance objectives, which again are predicted by personal determinants and environmental conditions. This is systematically shown in Figure 4. A fictitious example of a matrix of change objectives is shown in Table 1. For more inspiration, real world examples of matrices of change objectives are available in the literature (Dalum, Schaalma, \& Kok, 2012; Mikolajczak, Kok, \& Hospers, 2008).

With this information, you can consult overviews of behavior change methods/techniques, and match these to the relevant determinants. Documenting your choices and their justifications will allow you to clearly report the empirical and theoretical evidence for your intervention (Fuller, Pearson, \& Peters, 2013; Peters, Abraham, \& Crutzen, 2012; Schaalma \& Kok, 2009). In addition, by carefully planning your evaluation, this will allow you to pin-point opportunities for improvement, by making it easier to see where you may have omitted important beliefs (Bartholomew et al., 2011). Hopefully, the basic guidelines provided in this paper, combined with the free/cheap software suggestions in the appendix at http://osf.io/fp8kv, can contribute to making the mapping of determinants, beliefs, and environmental conditions more accessible. Note that the figures in this paper have been released into the public domain and are available at http://osf.io/fp8kv, so feel free to use these in training students or practitioners.

\section{References}

Alt, F. L. (1972). Archaelogy of computers: Reminiscences, 1945-1947. Communications of the ACM , 15(7), 693-694. doi:10.1145/361454.361528

Azevedo, F. a C., Carvalho, L. R. B., Grinberg, L. T., Farfel, J. M., Ferretti, R. E. L., Leite, R. E. P., ... Herculano-Houzel, S. (2009). Equal numbers of neuronal and nonneuronal cells make the human brain an isometrically scaled-up primate brain. The Journal of Comparative Neurology, 513(5), 532-541. doi:10.1002/cne.21974

Bandura, A. (1991). Social Cognitive Theory of SelfRegulation. Organizational Behavior and Human Decision Processes, 50(2), 248-287. doi:10.1016/0749-5978(91)90022-L

Bartholomew, L. K., Parcel, G. S., Kok, G., Gottlieb, N. H., \& Fernández, M. E. (2011). Planning health promotion programs: an Intervention Mapping approach (3rd ed.). San Francisco, CA: Jossey-Bass.

Dalum, P., Schaalma, H., \& Kok, G. (2012). The development of an adolescent smoking cessation intervention-an Intervention Mapping approach to planning. Health Education Research, 27(1), 172-181. doi:10.1093/her/cyr044

De Bruin, M., Viechtbauer, W., Hospers, H. J., Schaalma, H. P., \& Kok, G. (2009). Standard care quality determines treatment outcomes in control groups of HAART-adherence intervention studies: implications for the interpretation and comparison of intervention effects. Health Psychology, 28(6), 668-674. doi:10.1037/a0015989

Fishbein, M., \& Ajzen, I. (2010). Predicting and changing behavior: The reasoned action approach. New York: Psychology Press.

Francis, J. J., Eccles, M. P., Johnston, M., Walker, A., Grimshaw, J., Foy, R., ... Bonetti, D. (2004). Constructing questionnaires based on the theory of planned behaviour: A manual for health services 
researchers. Direct. Newcastle upon Tyne, UK. Retrieved from http://openaccess.city.ac.uk/1735/

Fuller, T. E., Pearson, M., \& Peters, J. L. (2013). Transparent reporting, the foundation for full disclosure. The European Health Psychologist, 15(3), 67-68. doi:10.2105/ajph.94.3.361

Gollwitzer, P. M. (1999). Implementation intentions: Strong effects of simple plans. The American Psychologist, 54(7), 493-503. doi:10.1037/0003066X.54.7.493

Hagger, M. S., \& Luszczynska, A. (2014). Planning interventions for behaviour change: A protocol for establishing best practice through consensus. The European Health Psychologist, 16(5), 206-213.

Khan, K. S., Kunz, R., Kleijnen, J., \& Antes, G. (2003). Five steps to conducting a systematic review. Journal of the Royal Society of Medicine, 96(3), 118-21.

Kok, G. (2014). A practical guide to effective behavior change: How to apply theory- and evidence-based behavior change methods in an intervention. The European Health Psychologist, 16(5), 156-170.

Kok, G., Gottlieb, N., Commers, M., \& Smerecnik, C. (2008). The ecological approach in health promotion programs: A decade later. American Journal of Health Promotion, 22(6), 437-442. doi:10.4278/ajhp.22.6.437

Mikolajczak, J., Kok, G., \& Hospers, H. J. (2008). Queermasters: developing a theory- and evidencebased internet hiv-prevention intervention to promote hiv-testing among men who have sex with men (MSM). Applied Psychology, 57(4), 681-697. doi:10.1111/j.1464-0597.2008.00342.x

Moher, D., Liberati, A., Tetzlaff, J., \& Altman, D. G. (2009). Preferred reporting items for systematic reviews and meta-analyses: the PRISMA statement. PLoS Medicine, 6(7), e1000097. doi:10.1371/journal.pmed.1000097

Peters, G.-J. Y. (2014). The alpha and the omega of scale reliability and validity: why and how to abandon Cronbach's alpha and the route towards more comprehensive assessment of scale quality. The European Health Psychologist, 16(2), 56-69.
Peters, G.-J. Y., Abraham, C. S., \& Crutzen, R. (2012). Full disclosure: doing behavioural science necessitates sharing. The European Health Psychologist, 14(4), 77-84.

Peters, G.-J. Y., de Bruin, M., \& Crutzen, R. (2014). Everything should be as simple as possible, but no simpler: towards a protocol for accumulating evidence regarding the active content of health behaviour change interventions. Health Psychology Review, 1-14. doi:10.1080/17437199.2013.848409

Peters, G.-J. Y., \& Kok, G. (2009). A structured review of reasons for ecstasy use and related behaviours: pointers for future research. BMC Public Health, 9, 230. doi:10.1186/1471-2458-9-230

Peters, G.-J. Y., Kok, G. J., \& Abraham, C. (2008). Social cognitive determinants of ecstasy use to target in evidence-based interventions: a metaanalytical review. Addiction, 103(1), 109-18. doi:10.1111/j.1360-0443.2007.02041.x

Ritchie, J., Lewis, J., McNaughton Nicholls, C., \& Ormston, R. (2014). Qualitative research practice: $A$ guide for Social Science students and researchers (2nd ed.). London: Sage Publications Ltd.

Roediger, H. L. I., \& McDermott, K. B. (1995). Creating false memories: Remembering words not presented in lists. Journal of Experimental Psychology: Learning, Memory and Cognition, 21(4), 803-814. doi:10.1037/0278-7393.21.4.803

Schaalma, H., \& Kok, G. (2009). Decoding health education interventions: The times are a-changin'. Psychology \& Health, 24(1), 5-9. doi:10.1080/08870440801995802

Schwarzer, R. (2008). Modeling health behavior change: How to predict and modify the adoption and maintenance of health behaviors. Applied Psychology: An International Review, 57(1), 1-29. doi:10.1111/j.1464-0597.2007.00325.x

Sniehotta, F. F., Schwarzer, R., Scholz, U., \& Schüz, B. (2005). Action planning and coping planning for long-term lifestyle change: theory and assessment. European Journal of Social Psychology, 35(4), 565-576. doi:10.1002/ejsp.258

Van Empelen, P., \& Kok, G. (2006). Condom use in steady and casual sexual relationships: Planning, 
preparation and willingness to take risks among adolescents. Psychology and Health, 21(2),

165-181. doi:10.1080/14768320500229898

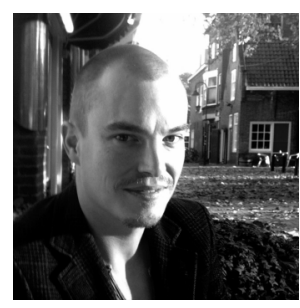

\section{Gjalt-J orn Y. Peters}

Open University of The Netherlands,

The Netherlands

gjalt-jorn@ behaviorchange.eu 